Теорія Ймовір. та Матем. Статист. Вип. 80, 2009
Theor. Probability and Math. Statist.

No. 80, 2010, Pages 119-130

S 0094-9000(2010)00799-X

Article electronically published on August 19, 2010

\title{
PATH PROPERTIES OF MULTIFRACTAL BROWNIAN MOTION
}

\author{
UDC 519.21
}

\author{
K. V. RAL'CHENKO AND G. M. SHEVCHENKO
}

\begin{abstract}
A new generalization of fractional Brownian motion (called multifractal Brownian motion) is considered for the case where the Hürst index $H$ is a function of time $t$. The pathwise continuity of multifractal Brownian motion is proved. Global and local Hölder properties are also studied.
\end{abstract}

\section{INTRODUCTION}

Fractional Brownian motion is used for modeling long-range dependence when studying processes in computer networks, in financial markets as well as in hydromechanics, climatology, and hydrography.

By definition, the fractional Brownian motion with Hürst index $H \in(0,1)$ is a Gaussian process $B^{H}=\left\{B_{t}^{H}, t \geq 0\right\}$ such that

(i) $B_{0}^{H}=0$;

(ii) $\mathrm{E} B_{t}^{H}=0, t \geq 0$;

(iii) $\mathrm{E}\left(B_{s}^{H} B_{t}^{H}\right)=\frac{1}{2}\left(s^{2 H}+t^{2 H}-|t-s|^{2 H}\right), s \geq 0, t \geq 0$.

The homogeneity of increments of fractional Brownian motion restricts substantially its applicability for modeling processes with long memory. In particular, it does not allow us to model processes whose regularity of paths and "memory depth" change in time. Several generalizations of fractional Brownian motion were proposed recently. A generalization, called multifractional Brownian motion, was proposed in the paper 8 . The definition in [8] is based on the Mandelbrot-van Ness representation. Namely, multifractional Brownian motion is defined by

$$
B_{H_{t}}(t)=\frac{1}{\Gamma\left(H_{t}+\frac{1}{2}\right)}\left\{\int_{-\infty}^{0}\left[(t-s)^{H_{t}-\frac{1}{2}}-(-s)^{H_{t}-\frac{1}{2}}\right] d W_{s}+\int_{0}^{t}(t-s)^{H_{t}-\frac{1}{2}} d W_{s}\right\}
$$

where $t \geq 0$ and $H:[0,+\infty) \rightarrow(0,1)$ is a Hölder function. If $H_{t} \equiv H$ is constant, the latter definition leads to the usual fractional Brownian motion. A generalization of this process to the case of a nonsmooth function $H_{t}$, called generalized multifractional Brownian motion, was proposed in the paper [1].

Introduced in [4, real harmonizable multifractional Lévy motion is defined as a realvalued random field admitting the following harmonizable representation:

$$
X_{h}(x)=\int_{\mathbb{R}^{d}} \frac{e^{-i x \cdot \xi}-1}{\|\xi\|^{d / 2+h(x)}} L(d \xi),
$$

2000 Mathematics Subject Classification. Primary 60G15, 60G17; Secondary 60G18.

Key words and phrases. Gaussian process, fractional Brownian motion, multifractal Brownian motion, Hürst index.

The authors are grateful to the European Commission for support of their investigations in the framework of the program "Marie Curie Actions", grant PIRSES-GA-2008-230804. 
where $L(d \xi)$ is a random Lévy measure and where $h(x)$ is a local Hölder function. If $L(d \xi)=W(d \xi)$ and $h$ is constant, then the latter definition gives multifractional Brownian motion. A similar generalization is considered in [2].

We propose below another generalization based on the representation of fractional Brownian motion with the help of Molchan's martingales (see, for example, [5, Chapter 1.8]).

Let a function $H:[0,+\infty) \rightarrow\left(\frac{1}{2}, 1\right)$ satisfy the Hölder condition with the exponent $\beta$, namely,

$$
\left|H_{t^{\prime}}-H_{t^{\prime \prime}}\right| \leq c\left|t^{\prime}-t^{\prime \prime}\right|^{\beta}
$$

for all $t^{\prime}, t^{\prime \prime} \in[0,+\infty)$ and for some $c>0$.

Definition 1. The stochastic process

$$
W_{t}^{H_{t}}=\int_{0}^{t} K_{H_{t}}(t, s) d W_{s}, \quad t \geq 0
$$

is called multifractal Brownian motion with a functional parameter $H$, where $W_{s}$ is a Wiener process,

$$
\begin{gathered}
K_{H_{t}}(t, s)=C_{H_{t}} s^{\frac{1}{2}-H_{t}} \int_{s}^{t}(v-s)^{H_{t}-\frac{3}{2}} v^{H_{t}-\frac{1}{2}} d v, \\
C_{H_{t}}=\left(\frac{H_{t}\left(2 H_{t}-1\right)}{\mathrm{B}\left(2-2 H_{t}, H_{t}-\frac{1}{2}\right)}\right)^{\frac{1}{2}} .
\end{gathered}
$$

If the function $H_{t}$ is constant, then relation (2) defines the usual fractional Brownian motion.

The paper is organized as follows. In Section 2, the continuity and Hölder property of trajectories of multifractal Brownian motion are proved. In Section 3 the local Hölder property of this process is studied. We show that the Hölder exponent of the multifractal Brownian motion at every point $t_{0} \geq 0$ equals $H_{t_{0}}$ almost surely.

\section{Properties of multifractal Brownian motion}

In this section, we study global continuity and the global Hölder property of paths of multifractal Brownian motion.

\subsection{Continuity.}

Theorem 1. The trajectories of the process $W_{t}^{H_{t}}$ are continuous with probability 1.

Proof. Put

$$
\begin{gathered}
\widetilde{K}_{H_{t}}(t, s)=s^{\frac{1}{2}-H_{t}} \int_{s}^{t}(v-s)^{H_{t}-\frac{3}{2}} v^{H_{t}-\frac{1}{2}} d v, \\
F(t)=\int_{0}^{t} \widetilde{K}_{H_{t}}(t, s) d W_{s} .
\end{gathered}
$$

Thus $W_{t}^{H_{t}}=C_{H_{t}} F(t)$. It is sufficient to prove that the stochastic process $F(t)$ is continuous with probability one in an arbitrary interval $[a, b] \subset[0,+\infty)$ such that $b-a<1$.

Note that

$$
\mathrm{E}\left(\left(F\left(t^{\prime}\right)-F\left(t^{\prime \prime}\right)\right)^{2}\right)=\mathrm{E}\left(\left(I_{1}+I_{2}+I_{3}\right)^{2}\right) \leq 3\left(\mathrm{E}\left(I_{1}^{2}\right)+\mathrm{E}\left(I_{2}^{2}\right)+\mathrm{E}\left(I_{3}^{2}\right)\right),
$$


where

$$
\begin{aligned}
& I_{1}=\int_{t^{\prime \prime}}^{t^{\prime}} \widetilde{K}_{H_{t^{\prime}}}\left(t^{\prime}, s\right) d W_{s}, \\
& I_{2}=\int_{0}^{t^{\prime \prime}}\left(\widetilde{K}_{H_{t^{\prime}}}\left(t^{\prime}, s\right)-\widetilde{K}_{H_{t^{\prime \prime}}}\left(t^{\prime}, s\right)\right) d W_{s}, \\
& I_{3}=\int_{0}^{t^{\prime \prime}}\left(\widetilde{K}_{H_{t^{\prime \prime}}}\left(t^{\prime}, s\right)-\widetilde{K}_{H_{t^{\prime \prime}}}\left(t^{\prime \prime}, s\right)\right) d W_{s} .
\end{aligned}
$$

Now we check the assumptions of the Kolmogorov continuity criterion for the process $F(t), t \in[a, b]$. Put $\mu:=\min _{t \in[a, b]} H_{t}$ and $\nu:=\max _{t \in[a, b]} H_{t}$. Without loss of generality, we assume that $a \leq t^{\prime \prime}<t^{\prime} \leq b$ and $\mu \leq H_{t^{\prime \prime}}<H_{t^{\prime}} \leq \nu$.

First we estimate $\mathrm{E}\left(I_{1}^{2}\right)$ :

$$
\begin{aligned}
& \mathrm{E}\left(I_{1}^{2}\right)=\int_{t^{\prime \prime}}^{t^{\prime}} \widetilde{K}_{H_{t^{\prime}}}^{2}\left(t^{\prime}, s\right) d s=\int_{t^{\prime \prime}}^{t^{\prime}} s^{1-2 H_{t^{\prime}}}\left(\int_{s}^{t^{\prime}}(v-s)^{H_{t^{\prime}}-\frac{3}{2}} v^{H_{t^{\prime}}-\frac{1}{2}} d v\right)^{2} d s \\
& \leq\left(t^{\prime}\right)^{2 H_{t^{\prime}}-1} \int_{t^{\prime \prime}}^{t^{\prime}} s^{1-2 H_{t^{\prime}}}\left(\int_{s}^{t^{\prime}}(v-s)^{H_{t^{\prime}}-\frac{3}{2}} d v\right)^{2} d s \\
& =\frac{\left(t^{\prime}\right)^{2 H_{t^{\prime}}-1}}{\left(H_{t^{\prime}}-\frac{1}{2}\right)^{2}} \int_{t^{\prime \prime}}^{t^{\prime}} s^{1-2 H_{t^{\prime}}}\left(t^{\prime}-s\right)^{2 H_{t^{\prime}}-1} d s \\
& \leq \frac{\left(t^{\prime}\right)^{2 H_{t^{\prime}}-1}}{\left(H_{t^{\prime}}-\frac{1}{2}\right)^{2}}\left(t^{\prime}-t^{\prime \prime}\right)^{2 H_{t^{\prime}}-1} \int_{t^{\prime \prime}}^{t^{\prime}} s^{1-2 H_{t^{\prime}}} d s \\
& \leq \frac{\left(t^{\prime}\right)^{2 H_{t^{\prime}}-1}\left(t^{\prime}\right)^{2-2 H_{t^{\prime}}}}{\left(H_{t^{\prime}}-\frac{1}{2}\right)^{2}\left(2-2 H_{t^{\prime}}\right)}\left(t^{\prime}-t^{\prime \prime}\right)^{2 H_{t^{\prime}}-1} \leq c_{1}\left(t^{\prime}-t^{\prime \prime}\right)^{2 \mu-1},
\end{aligned}
$$

where

$$
c_{1}=\frac{b}{\left(\mu-\frac{1}{2}\right)^{2}(2-2 \nu)} \text {. }
$$

Now we consider $\mathrm{E}\left(I_{2}^{2}\right)$ :

$$
\mathrm{E}\left(I_{2}^{2}\right)=\int_{0}^{t^{\prime \prime}}\left(\widetilde{K}_{H_{t}^{\prime}}\left(t^{\prime}, s\right)-\widetilde{K}_{H_{t}^{\prime \prime}}\left(t^{\prime}, s\right)\right)^{2} d s
$$

According to the Lagrange theorem, there exists $\tau \in\left[H_{t^{\prime \prime}}, H_{t^{\prime}}\right]$ such that

$$
\widetilde{K}_{H_{t}^{\prime}}\left(t^{\prime}, s\right)-\widetilde{K}_{H_{t}^{\prime \prime}}\left(t^{\prime}, s\right)=\left.\frac{\partial \widetilde{K}_{h}\left(t^{\prime}, s\right)}{\partial h}\right|_{h=\tau}\left(H_{t^{\prime}}-H_{t^{\prime \prime}}\right) .
$$

Thus,

$$
\begin{aligned}
\mathrm{E}\left(I_{2}^{2}\right) & =\int_{0}^{t^{\prime \prime}} s^{1-2 \tau}\left(\int_{s}^{t^{\prime}}(v-s)^{\tau-\frac{3}{2}} v^{\tau-\frac{1}{2}} \ln \frac{(v-s) v}{s} d v\right)^{2} d s \cdot\left(H_{t^{\prime}}-H_{t^{\prime \prime}}\right)^{2} \\
& \leq\left(t^{\prime}\right)^{\tau-\frac{1}{2}} \int_{0}^{t^{\prime \prime}} s^{1-2 \tau}\left(\int_{s}^{t^{\prime}}(v-s)^{\tau-\frac{3}{2}} \ln \frac{(v-s) v}{s} d v\right)^{2} d s \cdot\left(H_{t^{\prime}}-H_{t^{\prime \prime}}\right)^{2} .
\end{aligned}
$$


It is easily seen that, for all $\gamma>0$ and $\delta>0$, there are positive constants $C_{\gamma}$ and $\widetilde{C}_{\delta}$ such that

$$
\begin{gathered}
|\ln x| \leq C_{\gamma} x^{-\gamma} \quad \text { for } 0<x \leq 1 \\
|\ln x| \leq \widetilde{C}_{\delta} x^{\delta} \quad \text { for } x \geq 1
\end{gathered}
$$

Taking into consideration the relations $a \leq s \leq v \leq b$ and $b-a \leq 1$ and the latter inequalities we write

$$
\begin{gathered}
|\ln (v-s)| \leq C_{\gamma}(v-s)^{-\gamma}, \\
\left|\ln \frac{v}{s}\right| \leq \widetilde{C}_{\delta}\left(\frac{v}{s}\right)^{\delta} \leq \widetilde{C}_{\delta} b^{\delta} s^{-\delta} .
\end{gathered}
$$

Choosing $\gamma=(2 \mu-1) / 4$ and $\delta=(1-\nu) / 4$ we get

$$
\left|\ln \frac{(v-s) v}{s}\right| \leq c_{2}(v-s)^{\frac{1-2 \mu}{4}}+c_{3} s^{\frac{\nu-1}{4}} .
$$

Hence

$$
\mathrm{E}\left(I_{2}^{2}\right) \leq b^{\tau-\frac{1}{2}}\left(H_{t^{\prime}}-H_{t^{\prime \prime}}\right)^{2}\left(c_{2}^{2} A_{1}+c_{3}^{2} A_{2}\right)
$$

where

$$
\begin{aligned}
& A_{1}=\int_{0}^{t^{\prime \prime}} s^{1-2 \tau}\left(\int_{s}^{t^{\prime}}(v-s)^{\frac{4 \tau-2 \mu-5}{4}} d v\right)^{2} d s, \\
& A_{2}=\int_{0}^{t^{\prime \prime}} s^{\frac{3-8 \tau+\nu}{4}}\left(\int_{s}^{t^{\prime}}(v-s)^{\tau-\frac{3}{2}} d v\right)^{2} d s .
\end{aligned}
$$

Since $4 \tau-2 \mu-1 \geq 2 \mu-1>0$,

$$
\begin{aligned}
A_{1} & =\frac{16}{(4 \tau-2 \mu-1)^{2}} \int_{0}^{t^{\prime \prime}} s^{1-2 \tau}\left(t^{\prime}-s\right)^{\frac{4 \tau-2 \mu-1}{2}} d s \leq \frac{16\left(t^{\prime}\right)^{\frac{4 \tau-2 \mu-1}{2}}}{(4 \tau-2 \mu-1)^{2}} \int_{0}^{t^{\prime \prime}} s^{1-2 \tau} d s \\
& =\frac{16\left(t^{\prime}\right)^{\frac{4 \tau-2 \mu-1}{2}}\left(t^{\prime \prime}\right)^{2-2 \tau}}{(4 \tau-2 \mu-1)^{2}(2-2 \tau)} \leq \frac{8 b^{\frac{3-2 \mu}{2}}}{(2 \mu-1)^{2}(1-\nu)}=: c_{4}<+\infty .
\end{aligned}
$$

Since $7-8 \tau+\nu \geq 7-7 \nu>0$,

$$
\begin{aligned}
A_{2} & =\frac{4}{(2 \tau-1)^{2}} \int_{0}^{t^{\prime \prime}} s^{\frac{3-8 \tau+\nu}{4}}\left(t^{\prime}-s\right)^{2 \tau-1} d s \leq \frac{4\left(t^{\prime}\right)^{2 \tau-1}}{(2 \tau-1)^{2}} \int_{0}^{t^{\prime \prime}} s^{\frac{3-8 \tau+\nu}{4}} d s \\
& =\frac{4\left(t^{\prime}\right)^{2 \tau-1}}{(2 \tau-1)^{2}} \cdot \frac{4\left(t^{\prime \prime}\right)^{\frac{7-8 \tau+\nu}{4}}}{7-8 \tau+\nu} \leq \frac{16 b^{\frac{3+\nu}{4}}}{(2 \mu-1)^{2}(7-7 \nu)}=: c_{5}<+\infty .
\end{aligned}
$$

Therefore relations (1), (5), (6), and (7) imply that

$$
\mathrm{E}\left(I_{2}^{2}\right) \leq c_{6}\left(t^{\prime}-t^{\prime \prime}\right)^{2 \beta}
$$


Finally we estimate $\mathrm{E}\left(I_{3}^{2}\right)$ :

$$
\begin{aligned}
\mathrm{E}\left(I_{3}^{2}\right) & =\int_{0}^{t^{\prime \prime}}\left(\widetilde{K}_{H_{t^{\prime \prime}}}\left(t^{\prime}, s\right)-\widetilde{K}_{H_{t^{\prime \prime}}}\left(t^{\prime \prime}, s\right)\right)^{2} d s \\
& =\int_{0}^{t^{\prime \prime}}\left(s^{\frac{1}{2}-H_{t^{\prime \prime}}} \int_{t^{\prime \prime}}^{t^{\prime}}(v-s)^{H_{t^{\prime \prime}}-\frac{3}{2}} v^{H_{t^{\prime \prime}}-\frac{1}{2}} d v\right)^{2} d s \\
& \leq\left(t^{\prime}\right)^{2 H_{t^{\prime \prime}}-1} \int_{0}^{t^{\prime \prime}} s^{1-2 H_{t^{\prime \prime}}} d s\left(\int_{t^{\prime \prime}}^{t^{\prime}}\left(v-t^{\prime \prime}\right)^{H_{t^{\prime \prime}}-\frac{3}{2}} d v\right)^{2} \\
& =\frac{\left(t^{\prime}\right)^{2 H_{t^{\prime \prime}}-1}\left(t^{\prime \prime}\right)^{2-2 H_{t^{\prime \prime}}}}{\left(2-2 H_{t^{\prime \prime}}\right)\left(H_{t^{\prime \prime}}-\frac{1}{2}\right)^{2}}\left(t^{\prime}-t^{\prime \prime}\right)^{2 H_{t^{\prime \prime}}-1} \leq c_{7}\left(t^{\prime}-t^{\prime \prime}\right)^{2 \mu-1}
\end{aligned}
$$

where

$$
c_{7}=\frac{b}{(2-2 \nu)\left(\mu-\frac{1}{2}\right)^{2}} .
$$

Inequalities (3), (4), (8), and (9) yield

$$
\mathrm{E}\left(\left(F\left(t^{\prime}\right)-F\left(t^{\prime \prime}\right)\right)^{2}\right) \leq c_{8}\left(t^{\prime}-t^{\prime \prime}\right)^{2 \min \left(\beta, \mu-\frac{1}{2}\right)} .
$$

If $\xi \sim N\left(0, \sigma^{2}\right)$, then

$$
\mathrm{E}\left(|\xi|^{r}\right)=\frac{2^{r / 2} \Gamma\left(\frac{r+1}{2}\right)}{\Gamma\left(\frac{1}{2}\right)} \sigma^{r}
$$

for an arbitrary $r \geq 0$ (see, for example, [7, p. 110]). Therefore, for a given $\alpha>0$, there exists a constant $c_{9}>0$ such that

$$
\mathrm{E}\left(\left|F\left(t^{\prime}\right)-F\left(t^{\prime \prime}\right)\right|^{\alpha}\right) \leq c_{9}\left(t^{\prime}-t^{\prime \prime}\right)^{\alpha \cdot \min \left(\beta, \mu-\frac{1}{2}\right)} .
$$

Choosing $\alpha>\min ^{-1}\left(\beta, \mu-\frac{1}{2}\right)$, we derive from Kolmogorov's criterion that $F(t)$ is a continuous process in the interval $[a, b]$.

2.2. The Hölder property. Let $\left\{W_{t}^{H_{t}}, t \geq 0\right\}$ be a multifractal Brownian motion and $[a, b] \subset \mathbb{R}_{+}$. Put $\varkappa=\min \left(\beta, \min _{t \in[a, b]} H_{t}\right)$.

Theorem 2. For every $0<\varepsilon<\varkappa$, there exists a random variable $\eta>0$ such that $\mathrm{E}\left(|\eta|^{\gamma}\right)<+\infty$ for all $\gamma>0$ and

$$
\sup _{s, t \in[a, b]} \frac{\left|W_{s}^{H_{s}}-W_{t}^{H_{t}}\right|}{|s-t|^{\varepsilon}} \leq \eta .
$$

For the proof of the theorem, we need the following result (see [3]).

Lemma (Garsia-Rodemich-Rumsey inequality). Let $p>0, \alpha>\frac{1}{p}$, and let $f \in C([a, b])$. Then for all $s, t \in[a, b]$, there exists a constant $C_{\alpha, p}>0$ such that

$$
|f(t)-f(s)|^{p} \leq C_{\alpha, p}|t-s|^{\alpha p-1} \int_{a}^{b} \int_{a}^{b} \frac{|f(x)-f(y)|^{p}}{|x-y|^{\alpha p+1}} d x d y .
$$

Proof of Theorem 2, It is sufficient to prove the result for the case of $b-a \leq 1$. Putting $\alpha=(\varkappa+\varepsilon) / 2$ and $p=2 /(\varkappa-\varepsilon)$ in inequality (11) we obtain that

$$
\left|W_{s}^{H_{s}}-W_{t}^{H_{t}}\right| \leq C_{\varkappa, \varepsilon}|s-t|^{\varepsilon} \xi
$$


for all $s, t \in[a, b]$, where

$$
\xi=\left(\int_{a}^{b} \int_{a}^{b} \frac{\left|W_{x}^{H_{x}}-W_{y}^{H_{y}}\right|^{\frac{2}{\varkappa-\varepsilon}}}{|x-y|^{\frac{2 \varkappa}{\varkappa-\varepsilon}}} d x d y\right)^{\frac{\varkappa-\varepsilon}{2}} .
$$

Let $\gamma>2 /(\varkappa-\varepsilon)$. Then

$$
\mathrm{E}\left(|\xi|^{\gamma}\right) \leq\left(\int_{a}^{b} \int_{a}^{b} \frac{\left(\mathrm{E}\left(\left|W_{x}^{H_{x}}-W_{y}^{H_{y}}\right|^{\gamma}\right)\right)^{\frac{2}{\gamma(\varkappa-\varepsilon)}}}{|x-y|^{\frac{2 \varkappa}{\varkappa-\varepsilon}}} d x d y\right)^{\frac{\varkappa-\varepsilon}{2}} .
$$

Now we show that there exists a constant $C_{1}>0$ such that

$$
\mathrm{E}\left(\left|W_{x}^{H_{x}}-W_{y}^{H_{y}}\right|^{2}\right) \leq C_{1}|x-y|^{2 \varkappa} .
$$

If $H_{t}=$ const, then the process $W_{t}^{H_{t}}$ is a fractional Brownian motion. Thus

$$
\mathrm{E}\left(\left|W_{x}^{H_{x}}-W_{y}^{H_{x}}\right|^{2}\right)=|x-y|^{2 H_{x}} \leq|x-y|^{2 \varkappa} .
$$

Now we check that

$$
\mathrm{E}\left(\left|W_{y}^{H_{x}}-W_{y}^{H_{y}}\right|^{2}\right) \leq C_{2}\left|H_{x}-H_{y}\right|^{2} .
$$

We will use the notation $\mu, \nu$, and $\widetilde{K}$ introduced in the proof of Theorem 1 . There exists a number $\tau \in[\mu, \nu]$ such that

$$
\begin{aligned}
K_{H_{x}}(y, s)-K_{H_{y}}(y, s) & =\frac{\partial K_{\tau}(y, s)}{\partial \tau}\left(H_{x}-H_{y}\right) \\
& =\left(\frac{d C_{\tau}}{d \tau} \widetilde{K}_{\tau}(y, s)+C_{\tau} \frac{\partial \widetilde{K}_{\tau}(y, s)}{\partial \tau}\right)\left(H_{x}-H_{y}\right) .
\end{aligned}
$$

Thus

$$
\begin{aligned}
& \mathrm{E}\left(\left|W_{y}^{H_{x}}-W_{y}^{H_{y}}\right|^{2}\right)=\int_{0}^{y}\left(K_{H_{x}}(y, s)-K_{H_{y}}(y, s)\right)^{2} d s \\
& \quad \leq 2\left(H_{x}-H_{y}\right)^{2}\left(\left(\frac{d C_{\tau}}{d \tau}\right)^{2} \int_{0}^{y} \widetilde{K}_{\tau}^{2}(y, s) d s+C_{\tau}^{2} \int_{0}^{y}\left(\frac{\partial \widetilde{K}_{\tau}(y, s)}{\partial \tau}\right)^{2} d s\right) .
\end{aligned}
$$

The proof of the uniform boundedness of the integrals on the right hand side of the latter equality is the same as that in the proof of Theorem 1 . The beta function $\mathrm{B}(x, y)$ is positive and continuously differentiable for $(x, y) \in(0,+\infty)^{2}$. Thus the functions $C_{\tau}$ and $d C_{\tau} / d \tau$ are continuous for $\tau \in[\mu, \nu]$, whence we conclude that they are bounded in $[\mu, \nu]$. This completes the proof of inequality (16).

Considering inequality (1) we get

$$
\mathrm{E}\left(\left|W_{y}^{H_{x}}-W_{y}^{H_{y}}\right|^{2}\right) \leq C_{2}|x-y|^{2 \beta} \leq C_{2}|x-y|^{2 \varkappa} .
$$

The bounds (15) and (17) imply inequality (14), whence

$$
\mathrm{E}\left(\left|W_{x}^{H_{x}}-W_{y}^{H_{y}}\right|^{\gamma}\right) \leq C_{3}|x-y|^{\gamma \varkappa}
$$

for some $C_{3}>0$. Then we derive from inequality (13) that

$$
\mathrm{E}\left(|\xi|^{\gamma}\right) \leq C_{4}(b-a)^{\gamma(\varkappa-\varepsilon)}
$$

for $\gamma>2 /(\varkappa-\varepsilon)$. In view of Lyapunov's inequality, $\mathbf{E}\left(|\xi|^{\gamma}\right)<+\infty$ for all $\gamma>0$. 
Choosing $\eta=C_{\varkappa, \varepsilon} \xi$ we deduce (10) from (12).

\section{The LOCAL HÖLDER PROPERTY}

Throughout this section we assume that the function $H_{t}$ satisfies the following condition: for all $t \geq 0$,

$$
\frac{1}{2}<H_{t}<\min (1, \beta)
$$

Definition 2. The limit

$$
\varliminf_{h \rightarrow 0} \frac{\ln \left|f\left(t_{0}+h\right)-f\left(t_{0}\right)\right|}{\ln |h|}
$$

is called the Hölder exponent of the function $f$ at the point $t_{0}$.

This definition is equivalent to the definition of the Hölder exponent proposed in [8, 1]. Thus the following result holds.

Lemma. The Hölder exponent of a function $f$ at a point $t_{0}$ is equal to $H \in(0,1)$ if and only if the following two conditions hold:

1) for an arbitrary real number $\gamma$ such that $\gamma<H$,

$$
\lim _{h \rightarrow 0} \frac{\left|f\left(t_{0}+h\right)-f\left(t_{0}\right)\right|}{|h|^{\gamma}}=0 ;
$$

2) for an arbitrary real number $\gamma>H$,

$$
\varlimsup_{h \rightarrow 0} \frac{\left|f\left(t_{0}+h\right)-f\left(t_{0}\right)\right|}{|h|^{\gamma}}=+\infty .
$$

Proof. Necessity. Denote by $H$ the Hölder exponent of the function $f$ at a point $t_{0}$.

1. First let $\gamma<H$. Then

$$
\frac{\ln \left|f\left(t_{0}+h\right)-f\left(t_{0}\right)\right|}{\ln |h|}>\frac{\gamma+H}{2}
$$

for all sufficiently small $h$. Thus

$\ln \frac{\left|f\left(t_{0}+h\right)-f\left(t_{0}\right)\right|}{|h|^{\gamma}}=\ln \left|f\left(t_{0}+h\right)-f\left(t_{0}\right)\right|-\gamma \ln |h|<\frac{H-\gamma}{2} \ln |h| \rightarrow-\infty, \quad h \rightarrow 0$, whence (18) follows.

2. Now let $\gamma>H$. Then there exists a sequence $\left\{h_{n}, n \geq 1\right\}$ such that $h_{n} \rightarrow 0$ as $n \rightarrow \infty$ and

$$
\frac{\ln \left|f\left(t_{0}+h_{n}\right)-f\left(t_{0}\right)\right|}{\ln \left|h_{n}\right|}<\frac{\gamma+H}{2} .
$$

Thus

$$
\ln \frac{\left|f\left(t_{0}+h_{n}\right)-f\left(t_{0}\right)\right|}{\left|h_{n}\right|^{\gamma}}=\ln \left|f\left(t_{0}+h_{n}\right)-f\left(t_{0}\right)\right|-\gamma \ln \left|h_{n}\right|>\frac{H-\gamma}{2} \ln \left|h_{n}\right| \underset{n \rightarrow \infty}{\longrightarrow}+\infty
$$

for large $n$, whence equality (19) follows.

Sufficiency. We assume that conditions 1) and 2) of the lemma hold. Condition 1) implies that

$$
\frac{\ln \left|f\left(t_{0}+h\right)-f\left(t_{0}\right)\right|}{\ln |h|}=\frac{\ln \left|f\left(t_{0}+h\right)-f\left(t_{0}\right)\right|-\gamma \ln |h|}{\ln |h|}+\gamma>\gamma
$$

for all $\gamma<H$ and all sufficiently small $h$. Passing to the limit as $\gamma \rightarrow H$ we obtain

$$
\varliminf_{h \rightarrow 0} \frac{\ln \left|f\left(t_{0}+h\right)-f\left(t_{0}\right)\right|}{\ln |h|} \geq H .
$$


Finally we consider the case of $\gamma>H$. By condition 2), there exists a sequence of real numbers $\left\{h_{n}, n \geq 1\right\}$ such that $h_{n} \rightarrow 0$ as $n \rightarrow \infty$ and

$$
\frac{\ln \left|f\left(t_{0}+h_{n}\right)-f\left(t_{0}\right)\right|}{\ln \left|h_{n}\right|}=\frac{\ln \left|f\left(t_{0}+h_{n}\right)-f\left(t_{0}\right)\right|-\gamma \ln \left|h_{n}\right|}{\ln \left|h_{n}\right|}+\gamma<\gamma .
$$

Passing to the limit as $\gamma \rightarrow H$,

$$
\varliminf_{h \rightarrow 0} \frac{\ln \left|f\left(t_{0}+h\right)-f\left(t_{0}\right)\right|}{\ln |h|} \leq H .
$$

Thus the Hölder exponent of the function $f$ at the point $t_{0}$ is equal to $H$.

Theorem 3. The Hölder exponent of the multifractal Brownian motion $W_{t}^{H_{t}}$ at a point $t_{0} \geq 0$ is equal to $H_{t_{0}}$ with probability one.

Proof. Step 1. We evaluate the covariance function of the process $W_{t}^{H_{t}}$. For definiteness, let $s \leq t$. Put

$$
\psi\left(u, v_{1}, v_{2}\right)=u^{1-H_{s}-H_{t}} v_{1}^{H_{s}-\frac{1}{2}} v_{2}^{H_{t}-\frac{1}{2}}\left(v_{1}-u\right)^{H_{s}-\frac{3}{2}}\left(v_{2}-u\right)^{H_{t}-\frac{3}{2}} .
$$

Then

$$
\begin{aligned}
\mathrm{E}\left(W_{s}^{H_{s}} W_{t}^{H_{t}}\right) & =\int_{0}^{s} K_{H_{s}}(s, u) K_{H_{t}}(t, u) d u=C_{H_{s}} C_{H_{t}} \int_{0}^{s} \int_{u}^{s} \int_{u}^{t} \psi\left(u, v_{1}, v_{2}\right) d v_{2} d v_{1} d u \\
& =C_{H_{s}} C_{H_{t}} \int_{0}^{s} \int_{0}^{t} \int_{0}^{v_{1} \wedge v_{2}} \psi\left(u, v_{1}, v_{2}\right) d u d v_{2} d v_{1}=C_{H_{s}} C_{H_{t}}\left(I_{1}+I_{2}-I_{3}\right),
\end{aligned}
$$

where

$$
\begin{aligned}
& I_{1}=\int_{0}^{s} \int_{0}^{v_{1}} \int_{0}^{v_{2}} \psi\left(u, v_{1}, v_{2}\right) d u d v_{2} d v_{1}, \\
& I_{2}=\int_{0}^{t} \int_{v_{1}}^{t} \int_{0}^{v_{1}} \psi\left(u, v_{1}, v_{2}\right) d u d v_{2} d v_{1}, \\
& I_{3}=\int_{s}^{t} \int_{v_{1}}^{t} \int_{0}^{v_{1}} \psi\left(u, v_{1}, v_{2}\right) d u d v_{2} d v_{1} .
\end{aligned}
$$

Changing the variable $u=v_{2} z$, we obtain from [6. Lemma 2.2 (i)] that

$$
\begin{aligned}
\int_{0}^{v_{2}} & u^{1-H_{s}-H_{t}}\left(v_{1}-u\right)^{H_{s}-\frac{3}{2}}\left(v_{2}-u\right)^{H_{t}-\frac{3}{2}} d u \\
& =v_{2}^{-1} \int_{0}^{1} z^{1-H_{s}-H_{t}}(1-z)^{H_{t}-\frac{3}{2}}\left(\frac{v_{1}}{v_{2}}-z\right)^{H_{s}-\frac{3}{2}} d z \\
& =\mathrm{B}\left(2-H_{s}-H_{t}, H_{t}-\frac{1}{2}\right) v_{1}^{\frac{1}{2}-H_{t}} v_{2}^{\frac{1}{2}-H_{s}}\left(v_{2}-v_{1}\right)^{H_{s}+H_{t}-2}
\end{aligned}
$$

whence

$$
\begin{aligned}
I_{1} & =\mathrm{B}\left(2-H_{s}-H_{t}, H_{t}-\frac{1}{2}\right) \int_{0}^{s} \int_{0}^{v_{1}} v_{1}^{H_{s}-H_{t}} v_{2}^{H_{t}-H_{s}}\left(v_{2}-v_{1}\right)^{H_{s}+H_{t}-2} d v_{2} d v_{1} \\
& =\mathrm{B}\left(2-H_{s}-H_{t}, H_{t}-\frac{1}{2}\right) \mathrm{B}\left(1+H_{t}-H_{s}, H_{s}+H_{t}-1\right) \frac{s^{H_{s}+H_{t}}}{H_{s}+H_{t}},
\end{aligned}
$$


where the integral is evaluated with the help of the change of the variable $v_{2}=v_{1} x$. Analogously,

$$
\begin{aligned}
I_{2} & =\mathrm{B}\left(2-H_{s}-H_{t}, H_{s}-\frac{1}{2}\right) \int_{0}^{t} \int_{0}^{v_{2}} v_{1}^{H_{s}-H_{t}} v_{2}^{H_{t}-H_{s}}\left(v_{2}-v_{1}\right)^{H_{s}+H_{t}-2} d v_{2} d v_{1} \\
& =\mathrm{B}\left(2-H_{s}-H_{t}, H_{s}-\frac{1}{2}\right) \mathrm{B}\left(1+H_{s}-H_{t}, H_{s}+H_{t}-1\right) \frac{t^{H_{s}+H_{t}}}{H_{s}+H_{t}}, \\
& I_{3}=\mathrm{B}\left(2-H_{s}-H_{t}, H_{s}-\frac{1}{2}\right) \int_{s}^{t} \int_{v_{1}}^{t} v_{1}^{H_{s}-H_{t}} v_{2}^{H_{t}-H_{s}}\left(v_{2}-v_{1}\right)^{H_{s}+H_{t}-2} d v_{2} d v_{1} .
\end{aligned}
$$

Step 2. In what follows we assume that $s, t \in[a, b]$, where $0<a \leq t_{0} \leq b$ and $b-a<1$. We prove that there exists a constant $C>0$ such that

$$
\mathrm{E}\left(\left(W_{t}^{H_{t}}-W_{s}^{H_{s}}\right)^{2}\right) \geq C|t-s|^{H_{t}+H_{s}} .
$$

Taking into account the part of the theorem proved above, we conclude that

$$
\mathrm{E}\left(W_{t}^{H_{t}}\right)^{2}=t^{2 H} \text {. }
$$

Then

$$
\begin{gathered}
\mathrm{E}\left(\left(W_{t}^{H_{t}}-W_{s}^{H_{s}}\right)^{2}\right)=\mathrm{E}\left(W_{t}^{H_{t}}\right)^{2}+\mathrm{E}\left(W_{s}^{H_{s}}\right)^{2}-2 \mathrm{E}\left(W_{s}^{H_{s}} W_{t}^{H_{t}}\right) \\
\quad=2 C_{H_{t}} C_{H_{s}} I_{3}+\left(t^{2 H_{t}}-2 C_{H_{t}} C_{H_{s}} I_{2}\right)+\left(s^{2 H_{s}}-2 C_{H_{t}} C_{H_{s}} I_{1}\right) .
\end{gathered}
$$

Put

$$
f(t, H)=C_{H_{t}} C_{H} \mathrm{~B}\left(2-H-H_{t}, H-\frac{1}{2}\right) \mathrm{B}\left(1+H-H_{t}, H+H_{t}-1\right) \frac{t^{H}}{H+H_{t}} .
$$

It is not hard to prove that $f \in C^{2}([a, b] \times[\mu, \nu])$ (here, as above, we use the notation $\mu=\min _{t \in[a, b]} H_{t}$ and $\left.\nu=\max _{t \in[a, b]} H_{t}\right)$. Thus the derivatives $f_{H H}^{\prime \prime}\left(t, H_{t}\right)$ and $f_{H t}^{\prime \prime}\left(t, H_{t}\right)$ are bounded for $t \in[a, b]$.

Since $t^{2 H_{t}}=2 t^{H_{t}} f\left(t, H_{t}\right)$, we get

$$
\begin{aligned}
t^{2 H_{t}}-2 C_{H_{t}} C_{H_{s}} I_{2} & =2 t^{H_{t}}\left(f\left(t, H_{t}\right)-f\left(t, H_{s}\right)\right) \\
& =2 t^{H_{t}} f_{H}^{\prime}\left(t, H_{t}\right)\left(H_{t}-H_{s}\right)-t^{H_{t}} f_{H H}^{\prime \prime}(t, \theta)\left(H_{t}-H_{s}\right)^{2} \\
& =2 t^{H_{t}} f_{H}^{\prime}\left(t, H_{t}\right)\left(H_{t}-H_{s}\right)+o\left(|t-s|^{H_{t}+H_{s}}\right), \quad t \rightarrow s,
\end{aligned}
$$

in view of

$$
\left|t^{H_{t}} f_{H H}^{\prime \prime}(t, \theta)\left(H_{t}-H_{s}\right)^{2}\right| \leq C_{1}\left(H_{t}-H_{s}\right)^{2} \leq C_{1}|t-s|^{2 \beta}=o\left(|t-s|^{H_{t}+H_{s}}\right), \quad t \rightarrow s .
$$

Analogously,

$$
s^{2 H_{s}}-2 C_{H_{t}} C_{H_{s}} I_{1}=2 s^{H_{s}} f_{H}^{\prime}\left(s, H_{s}\right)\left(H_{s}-H_{t}\right)+o\left(|t-s|^{H_{t}+H_{s}}\right), \quad t \rightarrow s .
$$

Therefore,

$$
\begin{aligned}
\left(t^{2 H_{t}}\right. & \left.-2 C_{H_{t}} C_{H_{s}} I_{2}\right)+\left(s^{2 H_{s}}-2 C_{H_{t}} C_{H_{s}} I_{1}\right) \\
& =2\left(H_{t}-H_{s}\right)\left(t^{H_{t}} f_{H}^{\prime}\left(t, H_{t}\right)-s^{H_{s}} f_{H}^{\prime}\left(s, H_{s}\right)\right)+o\left(|t-s|^{H_{t}+H_{s}}\right) \\
& =2\left(H_{t}-H_{s}\right)\left(g\left(t, H_{t}\right)-g\left(s, H_{t}\right)+g\left(s, H_{t}\right)-g\left(s, H_{s}\right)\right)+o\left(|t-s|^{H_{t}+H_{s}}\right) \\
& =2\left(H_{t}-H_{s}\right)\left(g_{t}^{\prime}\left(\theta_{1}, H_{t}\right)(t-s)+g_{H}^{\prime}\left(s, \theta_{2}\right)\left(H_{t}-H_{s}\right)\right)+o\left(|t-s|^{H_{t}+H_{s}}\right), \quad t \rightarrow s,
\end{aligned}
$$


where $g(t, H)=t^{H} f_{H}^{\prime}(t, H)$. Since

$$
\begin{aligned}
\left|H_{t}-H_{s}\right| \cdot|t-s| \leq c|t-s|^{\beta+1}=o\left(|t-s|^{H_{t}+H_{s}}\right), \quad t \rightarrow s, \\
\left|H_{t}-H_{s}\right|^{2} \leq c^{2}|t-s|^{2 \beta}=o\left(|t-s|^{H_{t}+H_{s}}\right), \quad t \rightarrow s,
\end{aligned}
$$

we use the boundedness of the derivatives of the function $g$ for $t \in[a, b]$ and get

$$
\left(t^{2 H_{t}}-2 C_{H_{t}} C_{H_{s}} I_{2}\right)+\left(s^{2 H_{s}}-2 C_{H_{t}} C_{H_{s}} I_{1}\right)=o\left(|t-s|^{H_{t}+H_{s}}\right), \quad t \rightarrow s .
$$

By formula (22) we have

$$
\mathrm{E}\left(\left(W_{t}^{H_{t}}-W_{s}^{H_{s}}\right)^{2}\right)=2 C_{H_{t}} C_{H_{s}} I_{3}+o\left(|t-s|^{H_{t}+H_{s}}\right), \quad t \rightarrow s .
$$

Let us estimate $I_{3}$. Formula (20) implies that

$$
\begin{aligned}
I_{3} & \geq \mathrm{B}\left(2-H_{s}-H_{t}, H_{s}-\frac{1}{2}\right) \int_{s}^{t} \int_{v_{1}}^{t}\left(\frac{t}{s}\right)^{-|t-s|^{\beta}}\left(v_{2}-v_{1}\right)^{H_{s}+H_{t}-2} d v_{2} d v_{1} \\
& =\mathrm{B}\left(2-H_{s}-H_{t}, H_{s}-\frac{1}{2}\right)\left(\frac{t}{s}\right)^{-|t-s|^{\beta}} \cdot \frac{(t-s)^{H_{t}+H_{s}}}{\left(H_{t}+H_{s}-1\right)\left(H_{t}+H_{s}\right)} .
\end{aligned}
$$

Further, for $s, t \in[a, b]$,

$$
I_{3} \geq C_{2}(t-s)^{H_{t}+H_{s}}
$$

and (23) implies that

$\mathrm{E}\left(\left(W_{t}^{H_{t}}-W_{s}^{H_{s}}\right)^{2}\right) \geq C_{3}|t-s|^{H_{t}+H_{s}}+o\left(|t-s|^{H_{t}+H_{s}}\right) \geq C|t-s|^{H_{t}+H_{s}}, \quad t \rightarrow s$.

Step 3. For an arbitrary sequence of real numbers $\left\{h_{n}, n \geq 1\right\}$ such that $h_{n} \downarrow 0$ as $n \rightarrow \infty$ we prove that

$$
\varliminf_{n \rightarrow \infty} \frac{\ln \left|W_{t_{0}+h_{n}}^{H_{t_{0}+h_{n}}}-W_{t_{0}}^{H_{t_{0}}}\right|}{\ln \left|h_{n}\right|} \geq H_{t_{0}}
$$

almost surely. For a given $\varepsilon>0$, there exists an $N$ such that

$$
\min _{t \in\left[t_{0}, t_{0}+h_{N}\right]} H_{t}>H_{t_{0}}-\frac{\varepsilon}{2} \text {. }
$$

According to Theorem 2, there exists a random variable $\eta>0$ such that $\mathrm{E}\left(|\eta|^{\gamma}\right)<+\infty$ for all $\gamma>0$ and

$$
\sup _{s, t \in\left[t_{0}, t_{0}+h_{N}\right]} \frac{\left|W_{s}^{H_{s}}-W_{t}^{H_{t}}\right|}{|s-t|^{H_{t_{0}}-\varepsilon}} \leq \eta .
$$

This implies that

$$
\frac{\left|W_{t_{0}+h_{n}}^{H_{t_{0}+h_{n}}}-W_{t_{0}}^{H_{t_{0}}}\right|}{\left|h_{n}\right|^{H_{t_{0}}-\varepsilon}} \leq \eta
$$

for all $n \geq N$. Then, for $n \geq N$,

$$
\begin{aligned}
\frac{\ln \left|W_{t_{0}+h_{n}}^{H_{t_{0}+h_{n}}}-W_{t_{0}}^{H_{t_{0}}}\right|}{\ln \left|h_{n}\right|} & =\frac{\ln \left|W_{t_{0}+h_{n}}^{H_{t_{0}+h_{n}}}-W_{t_{0}}^{H_{t_{0}}}\right|}{\ln \left|h_{n}\right|} \\
& \geq \frac{\ln \eta+\ln \left|h_{n}\right|^{H_{t_{0}}-\varepsilon}}{\ln \left|h_{n}\right|}=\frac{\ln \eta}{\ln \left|h_{n}\right|}+H_{t_{0}}-\varepsilon \rightarrow H_{t_{0}}-\varepsilon, \quad n \rightarrow \infty .
\end{aligned}
$$

Passing to the limit as $\varepsilon \rightarrow 0$ we obtain the desired relation. 
Step 4. We prove that there exists a sequence $\left\{h_{n}, n \geq 1\right\}$ such that $h_{n} \rightarrow 0$ as $n \rightarrow \infty$ and

$$
\lim _{n \rightarrow \infty} \frac{\ln \left|W_{t_{0}+h_{n}}^{H_{t_{0}+h_{n}}}-W_{t_{0}}^{H_{t_{0}}}\right|}{\ln \left|h_{n}\right|}=H_{t_{0}}
$$

almost surely.

It is sufficient to prove that

$$
\frac{\ln \left|W_{t_{0}+h_{n}}^{H_{t_{0}+h_{n}}}-W_{t_{0}}^{H_{t_{0}}}\right|}{\ln \left|h_{n}\right|} \stackrel{\mathrm{P}}{\rightarrow} H_{t_{0}}, \quad n \rightarrow \infty,
$$

for an arbitrary sequence $\left\{h_{n}, n \geq 1\right\}$ such that $h_{n} \rightarrow 0$ as $n \rightarrow \infty$. In this case, there exists a subsequence for which (24) holds almost surely.

Using (21), we have

$$
\begin{aligned}
& \quad \mathrm{P}\left(\frac{\ln \left|W_{t_{0}+h_{n}}^{H_{t_{0}+h_{n}}}-W_{t_{0}}^{H_{t_{0}}}\right|}{\ln \left|h_{n}\right|}-H_{t_{0}}>\varepsilon\right) \\
& \quad=\mathrm{P}\left(\ln \left|W_{t_{0}+h_{n}}^{H_{t_{0}+h_{n}}}-W_{t_{0}}^{H_{t_{0}}}\right|-\ln \left|h_{n}\right|^{H_{t_{0}}}<\varepsilon \ln \left|h_{n}\right|\right) \\
& \quad=\mathrm{P}\left(\ln \left|W_{t_{0}+h_{n}}^{H_{t_{n}+h_{n}}}-W_{t_{0}}^{H_{t_{0}}}\right|<\ln \left|h_{n}\right|^{H_{t_{0}}+\varepsilon}\right) \\
& \quad=\mathrm{P}\left(\left|W_{t_{0}+h_{n}}^{H_{t_{0}+h_{n}}}-W_{t_{0}}^{H_{t_{0}}}\right|<\left|h_{n}\right|^{H_{t_{0}}+\varepsilon}\right) \\
& \quad \leq \mathrm{P}\left(\frac{\left|W_{t_{0}+h_{n}}^{H_{t+h_{n}}}-W_{t_{0}}^{H_{t_{0}}}\right|}{\sqrt{\mathrm{E}\left(\left(W_{t_{0}+h_{n}}^{H_{t_{0}+h_{n}}}-W_{t_{0}}^{H_{t_{0}}}\right)^{2}\right)}}<\frac{\left|h_{n}\right|^{H_{t_{0}}+\varepsilon}}{\sqrt{C\left|h_{n}\right|^{H_{t_{0}+h_{n}}+H_{t_{0}}}}}\right) \\
& \quad \leq \widetilde{C}\left|h_{n}\right|^{H_{t_{0}+\varepsilon-\frac{1}{2}\left(H_{t_{0}+h_{n}}+H_{t_{0}}\right)} \rightarrow 0,} n \rightarrow \infty,
\end{aligned}
$$

for sufficiently large $n$, since $H_{t_{0}}+\varepsilon-\frac{1}{2}\left(H_{t_{0}+h_{n}}+H_{t_{0}}\right)>0$ for sufficiently large integer numbers $n$.

The latter inequality holds, since

$$
\frac{\left|W_{t_{0}+h_{n}}^{H_{t_{0}+h_{n}}}-W_{t_{0}}^{H_{t_{0}}}\right|}{\sqrt{\mathrm{E}\left(\left(W_{t_{0}+h_{n}}^{H_{t_{0}+h_{n}}}-W_{t_{0}}^{H_{t_{0}}}\right)^{2}\right)}}
$$

is a standard normal random variable whose density is bounded.

\section{Concluding Remarks}

Multifractal Brownian motion is defined in the paper. This stochastic process generalizes the usual fractional Brownian motion to the case where its parameter $H$ (Hürst index) changes with time. It is shown that the trajectories of this process are continuous with probability one and satisfy the Hölder property in an arbitrary interval $[a, b]$. It is proved that the Hölder exponent at an arbitrary point $t_{0} \geq 0$ is equal to $H_{t_{0}}$ almost surely. 


\section{BIBLIOGRAPHY}

1. A. Ayache and J. Lévy Véhel, Generalized multifractional Brownian motion, Fractals: Theory and Applications in Engineering, Springer, London, 1999, pp. 17-32. MR1726365(2001d:60037)

2. J. M. Bardet and P. Bertran, Identification of the multiscale fractional Brownian motion with biomechanical applications, J. Time Series Anal. 28 (2007), no. 1, 1-52. MR2332850 (2008e:60095)

3. A. M. Garsia, E. Rodemich, and H. Rumsey, Jr., A real variable lemma and the continuity of paths of some Gaussian processes, Indiana Univ. Math. J. 20 (1970), no. 6, 565-578. MR 0267632(42:2534)

4. C. Lacaux, Real harmonizable multifractional Lévy motions, Ann. Inst. H. Poincaré Probab. Statist. 40 (2004), no. 3, 259-277. MR2060453 (2005b:60103)

5. Yu. Mishura, Stochastic Calculus for Fractional Brownian Motion and Related Processes, Lect. Notes Math., vol. 1929, Springer, Berlin, 2008. MR2378138 (2008m:60064)

6. I. Norros, E. Valkeila, and J. Virtamo, An elementary approach to a Girsanov formula and other analytical results on fractional Brownian motions, Bernoulli 5 (1999), no. 4, 571-587. MR:1704556 (2000f:60053)

7. A. Papoulis, Probability, Random Variables, and Stochastic Processes, 3rd ed., McGraw-Hill Book Company, New York, 1991. MR0176501 (31:773)

8. R. F. Peltier and J. Lévy Véhel, Multifractional Brownian Motion: Definition and Preliminary Results, INRIA research report, vol. 2645, 1995.

Department of Probability Theory, Statistics and Actuarial Mathematics, Faculty of Mechanics and Mathematics, Kyiv National Taras Shevchenko University, Volodymyrska 64, KYIV 01601, UKRAINE

E-mail address: k.ralchenko@gmail.com

Department of Probability Theory, Statistics and Actuarial Mathematics, Faculty of Mechanics and Mathematics, Kyiv National Taras Shevchenko University, Volodymyrska 64, KYIV 01601, UKRAINE

E-mail address: zhora@univ.kiev.ua

Received 12/MAR/2009

Translated by N. N. SEMENOV 